\title{
Texture Analysis for Classification of Endometrial Tissue in Gray Scale Transvaginal Ultrasonography
}

\author{
Anna Karahaliou ${ }^{1}$, Spyros Skiadopoulos ${ }^{1}$, George Michail ${ }^{2}$, Christina \\ Kalogeropoulou ${ }^{3}$, Ioannis Boniatis ${ }^{1}$, George Kourounis ${ }^{2}$, George \\ Panayiotakis ${ }^{1}$, and Lena Costaridou ${ }^{1}$ \\ 1 Department of Medical Physics, School of Medicine, University of \\ Patras \\ 2 Department of Obstetrics and Gynecology, School of Medicine, \\ University of Patras \\ 3 Department of Radiology, School of Medicine, University of Patras \\ costarid@upatras.gr
}

\begin{abstract}
Computer-aided classification of benign and malignant endometrial tissue, as depicted in 2D gray scale transvaginal ultrasonography (TVS), was attempted by computing texture-based features. 65 TVS endometrial images were collected ( 15 malignant, 50 benign) and processed with a wavelet based enhancement technique. Two regions of interest (ROIs) were identified (endometrium, endometrium margin) on each processed image. Thirty-two textural features were extracted from each ROI employing first and second order statistics texture analysis algorithms. Textural feature-based models were generated for differentiating benign from malignant endometrial tissue employing stepwise logistic regression analysis. Models' performance was evaluated by means of receiver operating characteristics (ROC) analysis. The best benign versus malignant classification was obtained from the model combining three textural features from endometrium and four textural features from endometrium margin, with corresponding area under $\mathrm{ROC}$ curve $(\mathrm{Az})$ 0.956 .
\end{abstract}

\section{Introduction}

Endometrial cancer is the fourth most prevalent malignant neoplasia among women [1]. The commonest presenting symptom of endometrial carcinoma is abnormal vaginal bleeding and thus is considered as indication for hysteroscopy and dilatation and curettage (D\&C), which is the 'golden standard' for histological evaluation. However, given that more than $90 \%$ of postmenopausal and more than $98 \%$ of preand perimenopausal women with abnormal vaginal bleeding will have a benign

Please use the following format when citing this chapter:

Karahaliou, Anna, Skiadopoulos, Spyros, Michail, George, Kalogeropoulou, Christina, Boniatis, Ioannis, Kourounis, George, Panayiotakis, George, Costaridou, Lena, 2006, in IFIP International Federation for Information Processing, Volume 204, Artificial Intelligence Applications and Innovations, eds. Maglogiannis, I., Karpouzis, K., Bramer, M., (Boston: Springer), pp. 713-721 
underlying cause, questions have arisen regarding the appropriateness of performing biopsies on all patients with bleeding [2].

Transvaginal sonographic assessment of the endometrium, which is usually based on endometrial thickness measurement, is a simple and non-invasive technique for excluding endometrial carcinoma. However, the cut-off values of endometrial thickness in literature vary considerably [3]. Most studies suggest the cut-off point of 4-5 mm endometrial thickness since it provides a high sensitivity, but specificity is reduced and thus resulting in many unnecessary biopsies.

Several approaches have been suggested in order to improve diagnostic accuracy of TVS. All of them focused on assessing qualitatively sonomorphological criteria (endometrial texture, regularity of endometrial-myometrial border, presence of central echo etc.) in addition to the sole measurement of endometrial thickness [4-6]. To the authors' knowledge, there are no reported studies assessing quantitatively endometrial texture for differentiating malignant from benign endometrial tissue. Computerized analysis of endometrial tissue (in terms of histogram based features) has only implemented in the context of ameliorating in vitro fertilization (IVF) outcome [7-9].

The aim of this study was to investigate the feasibility of computerized texture analysis in characterizing endometrial tissue as depicted in 2D gray scale TVS images. In particular, images were processed with a wavelet-based enhancement technique to facilitate delineation of two regions of interest (ROIs) corresponding to endometrium and to endometrium margin, respectively. First and second order textural features were extracted from the determined regions. Textural features-based models were generated for differentiating malignant from benign endometrial tissue, utilizing stepwise logistic regression analysis. Models' performance was evaluated by means of receiver operating characteristic (ROC) analysis.

\section{Materials and Methods}

\subsection{Subjects and Image Acquisition}

Eighty-two (82) perimenopausal and postmenopausal women with vaginal bleeding, scheduled for fractionated dilatation and curettage (D\&C) or hysterectomy in the Gynecological Department of the University Hospital of Patras, were recruited. Seventeen (17) patients were excluded from the study due to hormonal replacement therapy or tamoxifen medication, suboptimal visualization of the endometrium attributed to medioverted or retroverted uterus, cancelled surgery and conservative surgery-myectomy resulting in 65 remaining patients. The average age of the patients was 57.2 years (range: $41-80$ years). The histological diagnosis documented 15 malignant and 50 benign endometria.

All 65 patients underwent transvaginal scan on a single ATL HDI 3500 ultrasonic imager (Advanced Technology Laboratories, Bothell, WA, USA), with a 
multifrequency $\mathrm{C} 9-5 \mathrm{MHz}$ transvaginal transducer, 48 hours or less before scheduled surgery. An experienced radiologist performed the examinations and obtained multiple representative transverse and longitudinal images documenting endometrial thickness for each patient. The digital 2D B-mode transvaginal ultrasonic endometrial images had 8 bits pixel depth and $768 \times 576$ pixels resolution. A dataset of 65 longitudinal images, in DICOM format, were selected under the concurrence of a radiologist and a gynecologist. The relationship between endometrial thickness and histological diagnosis is presented graphically in Fig. 1. As expected, the greater the endometrial thickness the higher the incidence of endometrial cancer.

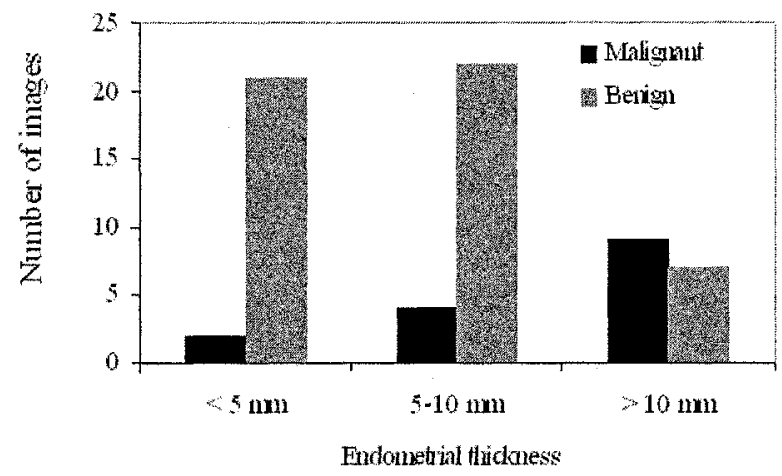

Fig. 1. Histogram illustrating the distribution of malignant and benign histological diagnosis with respect to endometrial thickness.

\subsection{Image Processing with a Wavelet-based Algorithm}

An image processing technique was employed to enhance the contrast of significant characteristics and to facilitate segmentation procedure. Each image of the dataset was processed with a locally adaptive wavelet based technique $[10,11]$. The method is based on local modification of multiscale gradient magnitude values provided by the redundant dyadic wavelet transform. Contrast enhancement is performed by applying a local linear mapping operator on multiscale gradient magnitude values. The first four frequency scales are used to enhance image characteristics of different size. Fig. 2(a) is an indicative image of the dataset, while Fig. 2(b) depicts the corresponding processed image acquired by application of the locally-adaptive wavelet technique.

An image visualization tool developed in our department $[12,13]$ has been used for application of the processing technique and the segmentation procedure described 
in the following section. This tool is domain-specific to medical imaging and provides global and adaptive wavelet functionality, in addition to conventional visualization operations.
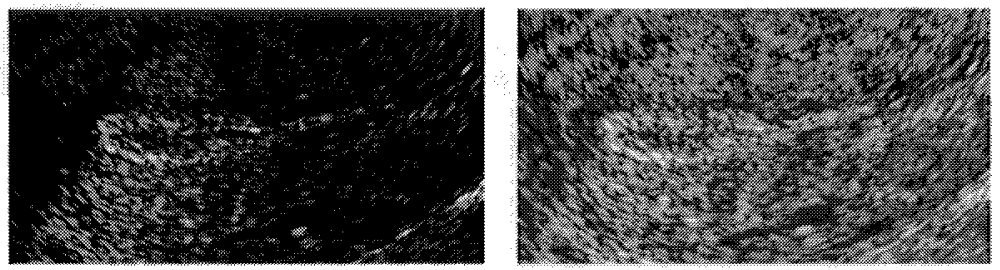

Fig. 2. (a) Indicative image of the dataset. (b) The corresponding processed image provided by application of the wavelet-based processing technique.

\subsection{Endometrial Tissue Segmentation}

A radiologist and a gynecologist, collaboratively, retraced manually a region of interest $\left(\mathrm{ROI}_{1}\right)$ corresponding to the endometrium without including endometrium border. A second region of interest $\left(\mathrm{ROI}_{2}\right)$ was delineated comprising of the endometrium along with the adjacent area of the myometrium (endometrium plus endometrium margin. To isolate the endometrium margin $\left(\mathrm{ROI}_{3}\right)$ a substraction of the two manually segmented ROIs was performed ( $\left.\mathrm{ROI}_{2}-\mathrm{ROI}_{1}\right)$. The two ROIs corresponding to endometrium ( $\left.\mathrm{ROI}_{1}\right)$ and endometrium margin $\left(\mathrm{ROI}_{3}\right)$ were used for the subsequent texture analysis. Figure 3 depicts the identified endometrium $\left(\mathrm{ROI}_{1}\right)$ and endometrium margin $\left(\mathrm{ROI}_{3}\right)$ on the processed image depicted in fig. 2(b).

\subsection{Feature Extraction}

Thirty-two (32) textural features were extracted from the endometrium ( $\left.\mathrm{ROI}_{1}\right)$ and endometrium margin $\left(\mathrm{ROI}_{3}\right)$ of each processed image, employing first and second order statistics texture analysis algorithms.

\subsubsection{First Order Statistics Textural Features}

First order statistics measure the likelihood of observing a specific gray level value at a randomly chosen location of the image. They are computed from the histogram of the image, and depend only on individual pixel values and not on the interaction or co-occurrence of neighboring pixel values. In this study, four first order textural features corresponding to the four moments of each ROI's gray level histogram were calculated: Mean value (MEA), Standard Deviation (SD), Skewness (SKEW) and Kurtosis (KURT). 


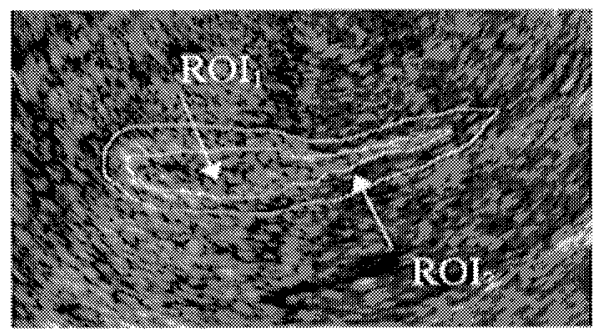

Fig. 3. Regions of interest corresponding to endometrium (ROI1) and to endometrium margin (ROI3).

\subsubsection{Second Order Statistics Textural Features}

The Gray Level Co-occurrence Matrix (GLCM) is a well-established robust statistical tool for extracting second order texture information from images [14,15]. The GLCM characterizes the spatial distribution of gray levels in the selected ROI. An element at location $(i, j)$ of the GLCM signifies the joint probability density of the occurrence of gray levels $i$ and $j$ in a specified orientation $\theta$ and specified distance $d$ from each other. In this study, four GLCMs corresponding to four different directions $\left(\theta=0^{\circ}, 45^{\circ}, 90^{\circ}\right.$ and $\left.135^{\circ}\right)$ and one distance $(d=1$ pixel), were computed for each selected ROI. Fourteen features were derived from each GLCM. Specifically, the features studied were: Angular Second Moment (ASM), Contrast (CON), Correlation (COR), Variance (VAR), Inverse Difference Moment (IDM), Sum Average (SUM_AV), Sum Variance (SUM_VAR), Sum Entropy (SUM_ENT), Entropy (ENT), Difference Entropy (DIF_ENT), Information Measure of Correlation 1 (IMCA1), Information Measure of Correlation 2 (IMCA2), Shade (SHA) and Promenance (PRO). Four values were obtained for each feature corresponding to the four matrices. The mean and range of these four values were calculated, comprising a total of twenty-eight second order textural features.

\subsection{Classification}

The 32 textural features extracted from endometrium ( $\left.\mathrm{ROI}_{1}\right)$ and endometrium margin $\left(\mathrm{ROI}_{3}\right)$ of the processed images were used as input variables for logistic regression analysis [16] to determine the subsets of features that can differentiate benign from malignant endometrial tissue. The histological findings regarding to benign or malignant diagnoses were considered as truth table. Specifically, the forward stepwise feature selection method of the logistic regression analysis was performed using a statistical software package (NCSS Statistical Software 2004, Kaysville, Utah, USA).

Two regression models were constructed, each one corresponding to endometrium $\left(\mathrm{ROI}_{1}\right)$ and to endometrium margin $\left(\mathrm{ROI}_{3}\right)$, respectively. Each model used as input variables the same 32 textural features extracted from $\mathrm{ROI}_{1}$ and $\mathrm{ROI}_{3}$, 
respectively. An additional regression model was constructed combining the 32 textural features extracted from $\mathrm{ROI}_{1}$ and the 32 textural features extracted from $\mathrm{ROI}_{3}$. This model will be referred as the one corresponding to $\left(\mathrm{ROI}_{1} / \mathrm{ROI}_{3}\right)$.

The best model for $\mathrm{ROI}_{1}, \mathrm{ROI}_{3}$ and $\mathrm{ROI}_{1} / \mathrm{ROI}_{3}$ was constructed in the form of $\operatorname{logit}(p)$ values as follows:

$$
\log i t(p)=\log _{e}\left(\frac{\operatorname{Prob}\left(Y=y_{1}\right)}{\operatorname{Prob}\left(Y=y_{2}\right)}\right)=\beta_{0}+\sum_{s=1}^{n} \beta_{s} X_{s}
$$

where $\mathrm{Xs}$ are the independent variables (features), $\mathrm{Y}$ is the binary dependent variable (pathology), which has two possible values, yl ( 0 : benign) and y2 (1: malignant), ßo is the intercept and $\beta$ s are the logistic regression coefficients. From these logit(p) values, the estimated probability of malignancy for a single case can be obtained from:

$$
p=\frac{\exp [\log i t(p)]}{1+\exp [\log i t(p)]}
$$

The leave-one-out validation methodology was performed for testing the logistic regression models. Their performance was evaluated by means of receiver operating characteristic (ROC) analysis, and classification accuracy was quantified by using the area under the ROC curve, $A_{z}$.

\section{Results}

Table 1. Best logistic regression models, as chosen by forward stepwise selection of textural features extracted from endometrium (ROI1), endometrium margin (ROI3), and combined endometrium/endometrium margin (ROI1/ROI3), in terms of logit(p) equations.

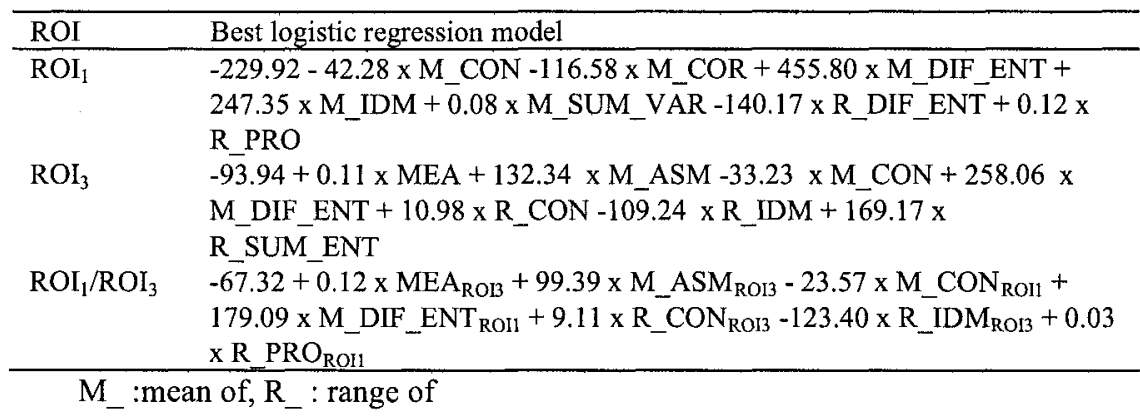




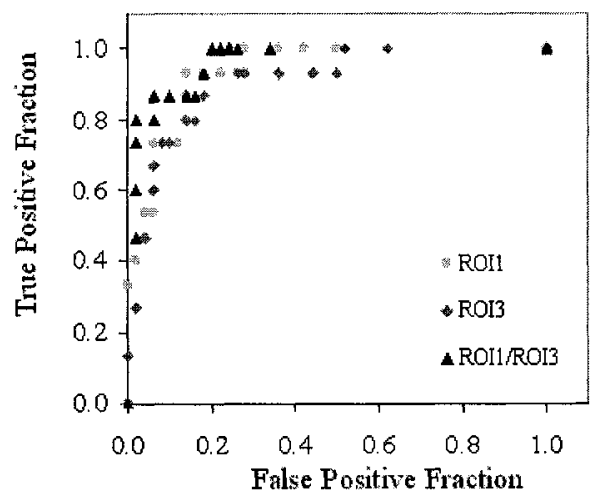

Fig. 4. ROC curves corresponding to the logistic regression feature-based models extracted from endometrium (ROI1), endometrium margin (ROI3) and combined endometrium/endometrium margin (ROI1/ROI3).

Table 1 provides the best logistic regression models, as chosen by forward stepwise selection of textural features extracted from $\mathrm{ROI}_{1}$ and $\mathrm{ROI}_{3}$, as well as for the model combining the features from $\mathrm{ROI}_{1}$ and $\mathrm{ROI}_{3}\left(\mathrm{ROI}_{1} / \mathrm{ROI}_{3}\right)$, in terms of logit $(p)$ equations. All features entered in the regression models are statistically significant (Wald-test for each regression coefficient, $p<0.05$ ). As observed in Table 1 , the features included in the regression models are differentiated with respect to the selected ROI.

The ROC curves corresponding to the three logistic regression models are presented in Fig. 4. The best performance is obtained by the model produced combining features from endometrium and endometrium margin ( $\mathrm{ROI}_{1} / \mathrm{ROI}_{3}$ ) achieving an $A_{z}$ value of 0.956 . The models corresponding to $\mathrm{ROI}_{1}$ and $\mathrm{ROI}_{3}$ achieved $A_{z}$ values of 0.937 and 0.909 , respectively.

\section{Discussion and Conclusion}

The feasibility of implementing computer-aided analysis using textural features to characterize endometrial tissue as depicted in 2D gray scale TVS was reported. The computer extracted textural features were selected, due to their proven applicability to analyze texture in ultrasound images [17-20]. A wavelet-based processing technique was employed to enhance the contrast of significant features in the image, and to identify ROIs from which textural features were extracted. Forward stepwise logistic regression analysis was then implemented to determine the best combination of textural features and to characterize endometrial tissue.

Both endometrium and endometrium margin seem to possess significant textural information that can efficiently distinguish malignant from benign endometrium 
tissue. However, the combination of textural features from both regions yields the best classification performance.

It was suggested that the computer aided classification of the endometrial tissue, based on texture-based features, can contribute to a correct diagnosis of endometrial malignancy and thereby potentially help reduce unnecessary biopsies.

However, the reported results are preliminary and additional work is required to improve performance of the proposed method. Future efforts will focus on validating the proposed method using a larger dataset and by examining cases of blind histology. Future work will focus on investigating the potential increase in the diagnostic accuracy of TVS by combining computerized textural features with orientation features and by using other classification methods.

\section{Acknowledgements}

The authors would like to thank the staff of the Department of Radiology at the University Hospital of Patras for their contribution in this work.

\section{References}

1. S.L. Parker, T. Tong, S. Bolden, P.A. Wingo, Cancer Statistics, CA Cancer J. Clin. 46, 527 (1996).

2. T.J. Dubinsky, Value of Sonography in the diagnosis of abnormal vaginal bleeding, $J$. Clin. Ultras. 32, 348-353 (2004).

3. J.K. Gupta, P.F.W. Chien, D. Voit, T.J. Clark, K.S. Khan, Ultrasonographic endometrial thickness for diagnosing endometrial pathology in women with postmenopausal bleeding: a meta-analysis, Acta Obstetr. et Gynec. Scand. 81, 799-816 (2002).

4. M. Weigel, K. Friese, F. Strittmatter, F. Melchert, Measuring the thickness-Is that all we have to do for sonographic assessment of endometrium in postmenopausal women? Ultras. Obstet. Gynecol. 6 97-102 (1995).

5. G. Weber, E. Merz, F. Bahlmann, B. Rösch, Evaluation of different transvaginal sonographic diagnostic parameters in women with postmenopausal bleeding, Ultras. Obstet. Gynecol. 12, 265-270 (1998).

6. B. Randelzhofer, H.J. Prompler, W. Sauerbrei, H. Madjar, G. Emons, Value of sonomorphologic criteria of the endometrium in women with postmenopausal bleeding: a multivariate analysis, Ultrasound. Obstet. Gynecol. 19, 62-68 (2002).

7. Z. Leibovitz, S. Degani, R. Rabia, J. Tal, I. Eibchitz, I. Shapiro, Y. Paltieli, A. Aharoni, M. Steinkuler, G. Ohel, Endometrium-to-myometrium relative echogenicity coefficient. A new sonographic approach for the quantitative assessment of endometrial echogenicity. Gynecol. Obstet. Invest. 45, 121-125 (1998).

8. R. Fanchin, C. Righini, J.M. Ayoubi, F. Olivennes, D. de Ziegler, R. Frydman, New look at the endometrial echogenicity: objective computer-assisted measurements predict endometrial receptivity in vitro fertilization - embryo transfer, Fertil. Steril. 74, 274-280 (2000).

9. C.H. Yang, P.C. Chung, Y.C. Tsai, Endometrium estimation in a sequence of ultrasonic images, Comput. Med. Imag. Graph. 25, 335-342 (2001). 
10.P. Sakellaropoulos, L. Costaridou, G. Panayiotakis, A wavelet-based spatially adaptive method for mammographic contrast enhancement, Phys. Med. Biol. 48, 787-803 (2003).

11.L. Costaridou, P. Sakellaropoulos, S. Skiadopoulos, G. Panayiotakis, Locally adaptive wavelet contrast enhancement. In: Medical Image Analysis Methods, edited by L. Costaridou (Taylor \& Francis Group LCC, CRC Press: Boca Raton, FL, 2005), pp. 225270.

12.P. Sakellaropoulos, L. Costaridou, G. Panayiotakis, An image visualization tool in mammography, Med. Inform. 24, 53-73 (1999).

13.P. Sakellaropoulos, L. Costaridou, G. Panayiotakis, Using component technologies for web based wavelet enhanced mammographic image visualization, Med. Inform. 25, 171-181 (2000).

14.R.M. Haralick, K. Shanmugam, I. Dinstein, Textural features for image classification, IEEE Trans. Syst. Man. Cybern. 3, 610-621 (1973).

15.R.F. Walker, P. Jackway, I.D. Longstaff, Improving Co-occurrence Matrix Feature Discrimination, in: Proc, of the $3^{\text {rd }}$ Conference on Digital Image Computing: Techniques and Applications, 643-648 (1995).

16.S. Menard, Applied Logistic Regression Analysis (Sage Publications, Thousand Oaks, CA, 2002).

17.H. Yoshida, D.D. Casalino, B. Keserci, A. Coskun, O. Ozturk, A. Savranlar, Waveletpacket-based texture analysis for differentiation between benign and malignant liver tumours in ultrasound images, Phys. Med. Biol. 48, 3735-3753 (2003).

18.R. Sivaramakrishna, K.A. Powell, M.L. Lieber, W.A. Chilcote, R. Shekhar, Texture analysis of lesions in breast ultrasound images, Comput. Med. Imag. Graph. 26, 303-307 (2002).

19.D. Smutek, R. Sara, P. Sucharda, T. Tjahjadi, M. Svec, Image texture analysis of sonograms in chronic inflammations of thyroid gland, Ultras. Med. Biol. 29, 1531-1543 (2003).

20.C.I. Christodoulou, C.S. Pattichis, E. Kyriacou, M.S. Pattichis, M. Pantziaris, A. Nicolaides, Texture and morphological analysis of ultrasound images of the carotid plaque for the assessment of stroke, in: Medical Image Analysis Methods, edited by L. Costaridou (Taylor \& Francis Group LLC, CRC Press: Boca Raton, FL, 2005), pp. 87-135. 